\title{
Conservative Management could be an Successful Option even in Severe Class III Emphysematous Pyelonephritis: Case Report
}

\author{
Keon-Cheol Lee \\ Department of Urology, Inje University,Ilsanpaik Hospital, Goyang, Korea
}

\begin{abstract}
Emphysematous pyelonephritis (EPN) is a rare infectious renal disease destructing renal parenchyme severely with acute clinical course. With the aid of percutaneous drainage and improved clinical care, the conservative treatment substituted nephrectomy in mild forms of EPN. But, in severe EPN with wide destructive necrotic lesions, nephrectomy is thought as inevitable. Here, we present our recent experience of successful conservative management of class III severe EPN patient with related literature review.
\end{abstract}

Keywords: Pyelonephritis;Emphysema;Conservative Treatment

\section{INTRODUCTION}

Emphysematous pyelonephritis (EPN) is a rare infectious renal disease destructing renal parenchyme severely with acute clinical course. For a long time after the naming of this serious disease by Schultz and Klorfein, it has a high mortality rate necessitating early emergency nephrectomy as a gold standard treatment [1].With the advent of CT and improved conservatie management strategy, successful conservative treatment could be possible in mild forms of selected EPN cases. And conservative management is being perfomed in more cases rather than nephrectomy with time passed [2]. With the aid of percutaneous drainage, the treatment outcomes also improved in EPN [3]. But, in severe EPN with wide destructive necrotic lesions, nephrectomy is thought as inevitable. Here, we present our recent experience of successful conservative management of class III severe EPN patient with related literature review.

\section{CASE REPORT}

A 56 years old female visited emergency department with a chief complaint of fever, chilling. Her underlying diseases were diabetes mellitus and chronic kidney disease although she had not received dialysis yet. She had weight loss during the last one month and since one week ago, had high fever, chilling and abdominal pain. Mental status was alert and chest breathing sound was clear and normoactive bowel sound was checked. Initial blood pressure was low as 93/62 $\mathrm{mmHg}$ and she had dehydrated tongue and skin turgor. Body temperature was $38.9{ }^{\circ} \mathrm{C}$ and pulse rate was $121 / \mathrm{min}$ and respiratory rate was $18 / \mathrm{min}$. Leukocyte was $22,180 / \mathrm{uL}$ and CRP was $46.0 \mathrm{mg} / \mathrm{dL}$ and sodium was $118 \mathrm{mEq} / \mathrm{L}$ and D-Dimer was $7.46 \mu \mathrm{g} / \mathrm{mL}$.On physical examination, severe left costovertebral angle tenderness (CVAT) was noted. On enhanced CT scan, severe destructive lesions were found in left kidney (Fig. 1). After diagnosing as class 3emphysematous pyelonephritis (EPN), we recommend emergent nephrectomy to her but she refused any operation. We considered her as septic condition patient and admitted her to the intensive care unit with close monitoring of vital sign. Urine and blood culture result was Esherichia coli with resistance to ampicillin, gentamicin and trimethoprim/sulfamethoxazole only. Antibiotic coverge was performed to gram positive, negative and even anarobes although other organisms was not cultured. After 4days later, the vital sign became stable and the patient regained oral intake and she agreed to perform the percutaneous catheter drainage. The patient recovered slowly and then 2 weeks later, she discharged from the hospital. Follow up CT scan was perfomed in 1 and 3 months later with near complete resolution of the lesion. (Fig. 2, 3). Now she had intermittent vague left flank discomfort without definite abnormalities. 
Conservative Management could be an Successful Option even in Severe Class III Emphysematous Pyelonephritis: Case Report

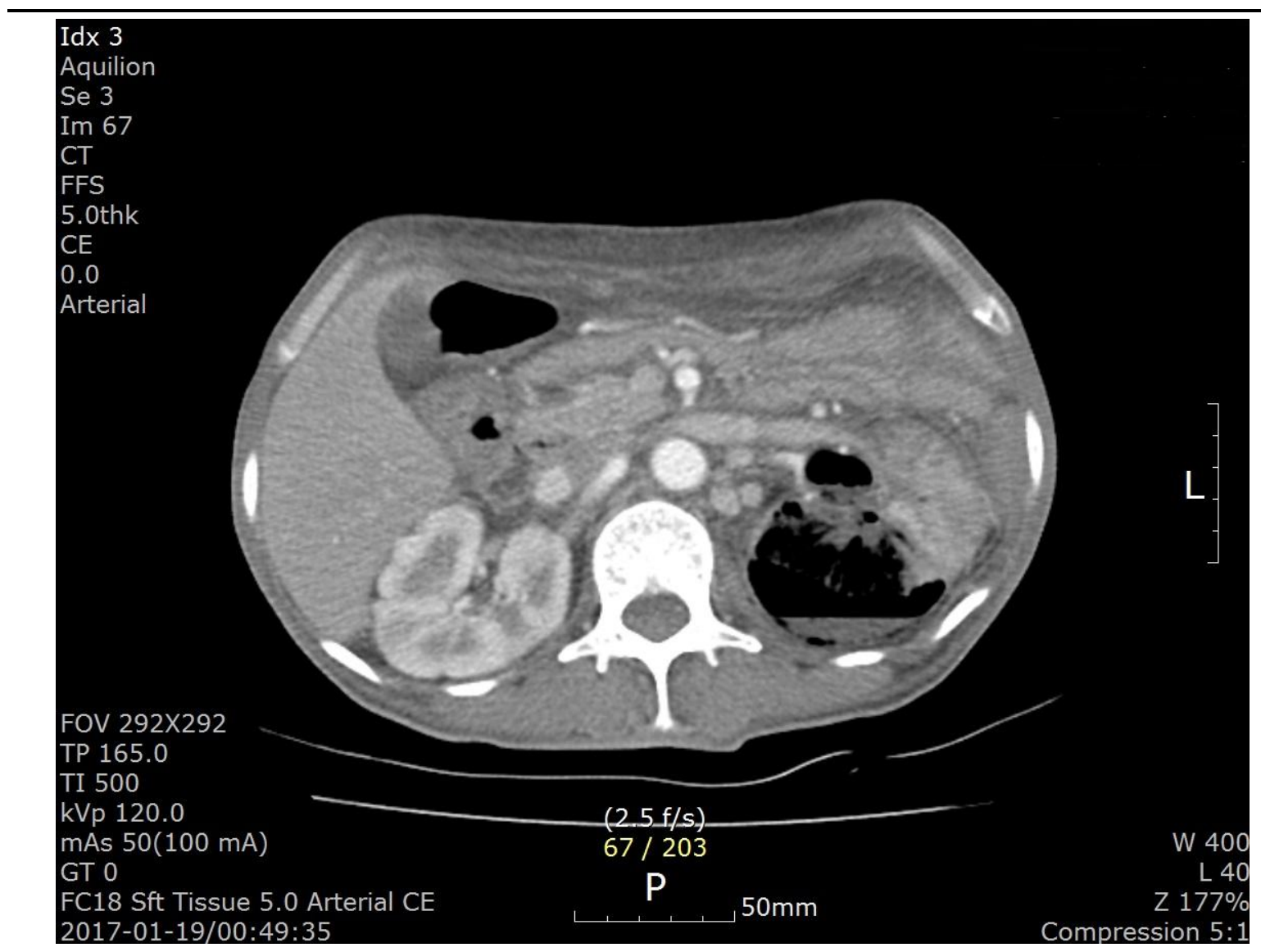

Fig1. Contrast enhanced CT scan showing large air-fluid lesion with infiltrating peri-renal space of necrotizing inflammation.

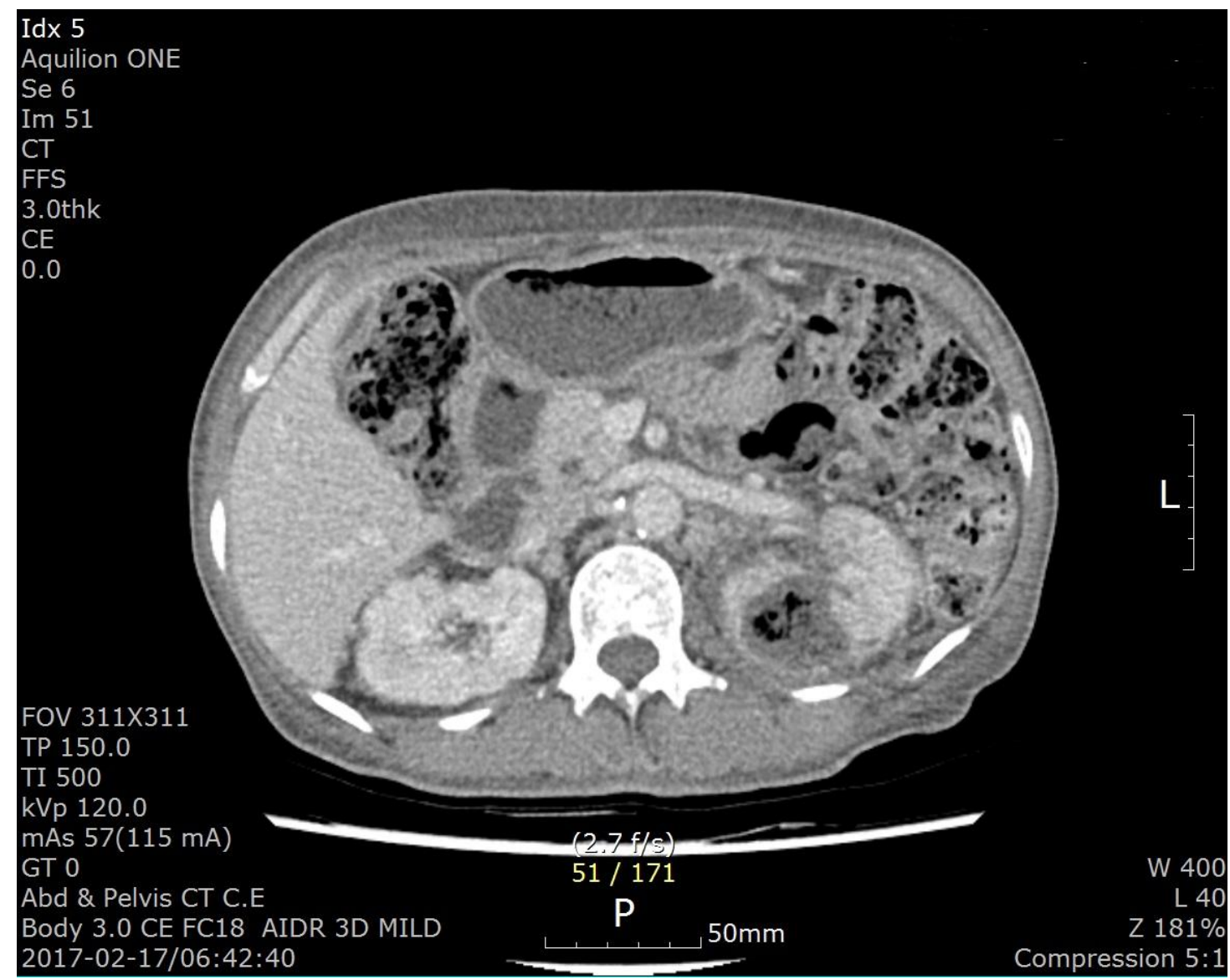

Fig2. Much improved destructed renal parenchymal lesions could be seen after 1 month follow up CT scan. 


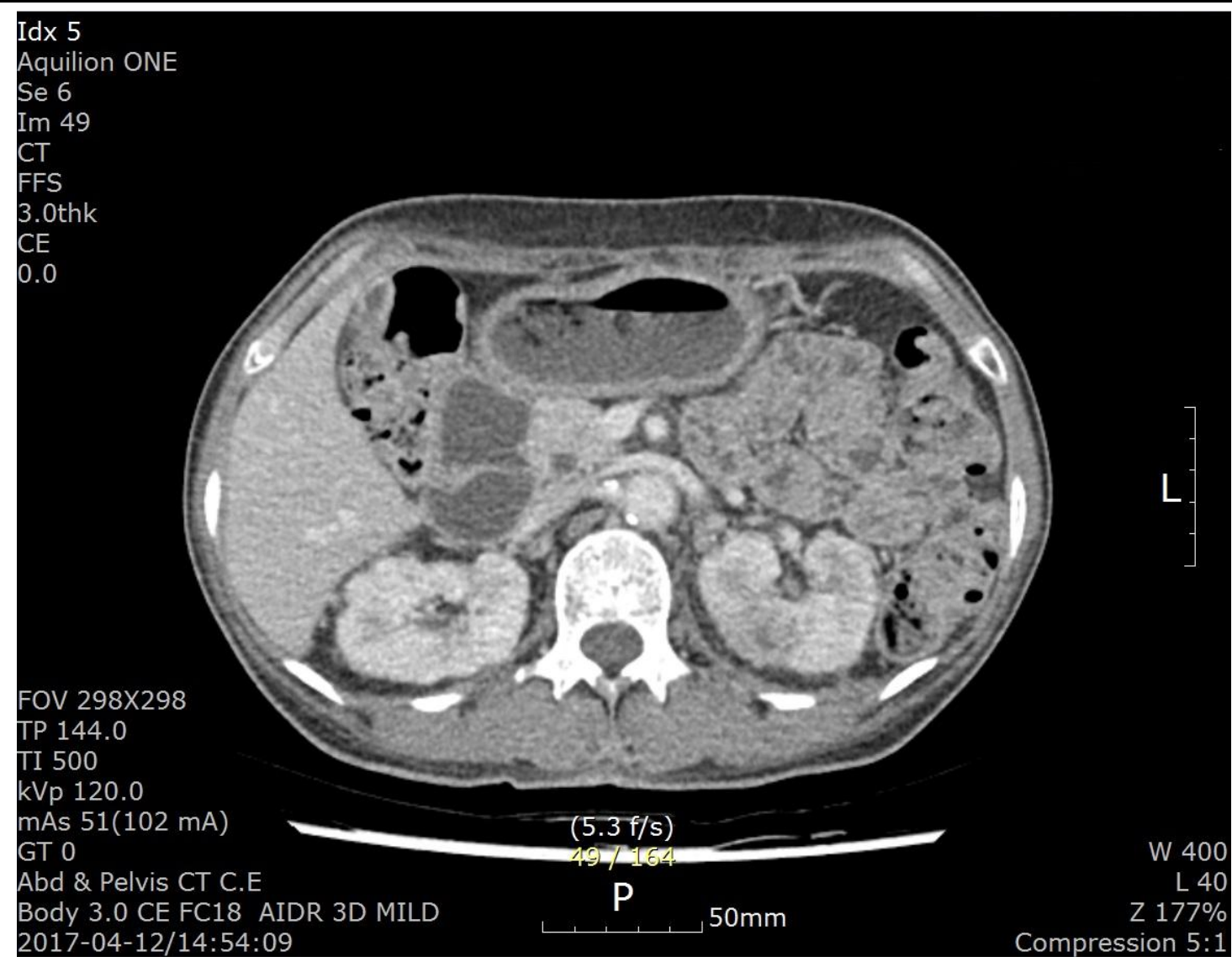

Fig. 3.Near complete resoultion of the lesions could be seen after 3 months later follow up CT scan.

\section{DISCUSSION}

Most common predisposing factor of EPN is diebetes mellitus and followed by urinary tract obstruction [4]. Our patient was also diabetes although she had no obstruction of urinary tract.Poor prognostic factors was known as severe leukocytosis, thrombocytopenia and radiologic class 3 with significant adverse outcomes. In other study, higher mortality rate was associated with altered mental status, thrombocytopenia, renal failure and severe hyponatremia at presentation [5]. In this case report, the patient was chronic renal failure although she was not receiving dialysis yet.

There are several classification systems of EPN but the most commonly used one is Huang and Tseng classification [6]. They classified EPN as 4 categories. Class I is EPN with gas in the collecting system only and class II one with gas in the renal parenchyme. In class III-A, gas or abscess extends into the perirenal space and III-B has gas in the pararenal space. Our patient was III-A or III-B with the most severe form of EPN. Class IV is bilateral or single kidney EPN.

Emphysematous pyelitis is the form of EPN with gas present in the collecting system only which is distinct from EPN [7]. In H\&T classification, it is class I EPN. Our patient has also air full-filled pelvocalyceal system besides the destructive renal parenchymal lesions. Gas in the collecting system has a good prognosis compared with one of renal parenchyme.

Escherichia coli is the most common pathogen in EPN and our patient also showed E. coli in blood and urine culture with favorable antibiotic sensitivity result. But, we used 3 different antibiotics to cover gram positive, negative and anarobes because there could be non-cultured organisms.

In conclusions, severe class 3 EPN could be treated with nephrectomy in first option, but, in selected patients conservative management could be an option with sucessful outcomes.

\section{REFERENCES}

[1] Schultz EH Jr, Klorfein EH. Emphysematous pyelonephritis. J Urol. 1962;87:762-6

[2] Sokhal AK, Kumar M, Purkait B, Jhanwar A, Singh K, Bansal A, et al. Emphysematous pyelonephritis: Changing trend of clinical spectrum, pathogenesis, management and outcome. Turk J Urol. 2017;43(2):202-9 
Conservative Management could be an Successful Option even in Severe Class III Emphysematous Pyelonephritis: Case Report

[3] Chen MT, Huang CN, Chou YH, et al. Percutaneous drainage in the treatment of emphysematous pyelonephritis: 10-year experience. J Urol. 1997;157:1569-73

[4] Ubee SS, McGlynn L, Fordham M. Emphysematous pyelonephritis.BJU Int. 2011;107(9):1474-8

[5] Kapoor R, Muruganandham K, Gulia AK, Singla M, Agrawal S, Mandhani A, et al. Predictive factors for mortality and need for nephrectomy in patients with emphysematous pyelonephritis. BJU Int. 2010;105(7):986-9

[6] Huang JJ, Tseng CC. Emphysematous pyelonephritis: clinicoradiological classification, management, prognosis, and pathogenesis. Arch Intern Med. 2000;27;160(6):797-805

[7] Maiti A, Saha D, Das A. Emphysematous Pyelitis: An Entity Distinct from Emphysematous Pyelonephritis. Am J Med Sci. 2017;353(5):505.

Citation: Keon-Cheol Lee. Clinical Conservative Management could be an Successful Option even in Severe Class III Emphysematous Pyelonephritis: Case Report.ARC Journal of Urology. 2017; 2(1):18-21.

Copyright: () 2017 Keon-Cheol Lee. This is an open-access article distributed under the terms of the Creative Commons Attribution License, which permits unrestricted use, distribution, and reproduction in any medium, provided the original author and source are credited. 